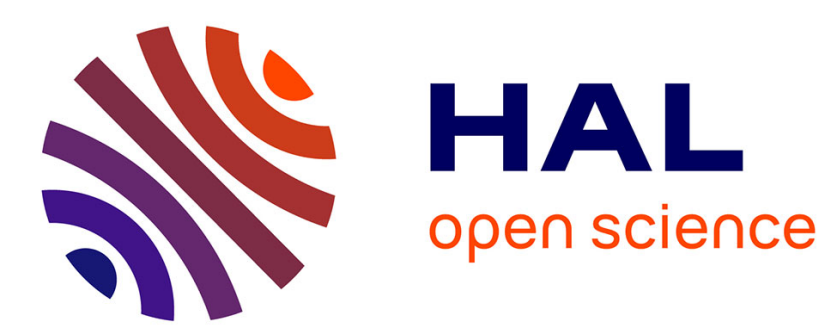

\title{
RÉUSSIR DANS LES PECO EN INTRODUISANT L'ORIENTATION MARCHE: Une réflexion à partir du cas roumain
}

Corinne Rochette

\section{To cite this version:}

Corinne Rochette. RÉUSSIR DANS LES PECO EN INTRODUISANT L'ORIENTATION MARCHE:

Une réflexion à partir du cas roumain. Décisions Marketing, 2006. hal-02884564

\section{HAL Id: hal-02884564 \\ https://hal.science/hal-02884564}

Submitted on 29 Jun 2020

HAL is a multi-disciplinary open access archive for the deposit and dissemination of scientific research documents, whether they are published or not. The documents may come from teaching and research institutions in France or abroad, or from public or private research centers.
L'archive ouverte pluridisciplinaire HAL, est destinée au dépôt et à la diffusion de documents scientifiques de niveau recherche, publiés ou non, émanant des établissements d'enseignement et de recherche français ou étrangers, des laboratoires publics ou privés. 
RÉUSSIR DANS LES PECO EN INTRODUISANT L'ORIENTATION MARCHÉ : Une réflexion à partir du cas roumain

Author(s): CORINNE ROCHETTE

Source: Décisions Marketing, Juillet-Décembre 2006, No. 43/44, SPÉCIAL

INTERNATIONAL (Juillet-Décembre 2006), pp. 147-159

Published by: Association Française du Marketing

Stable URL: http://www.jstor.com/stable/40593087

\section{REFERENCES}

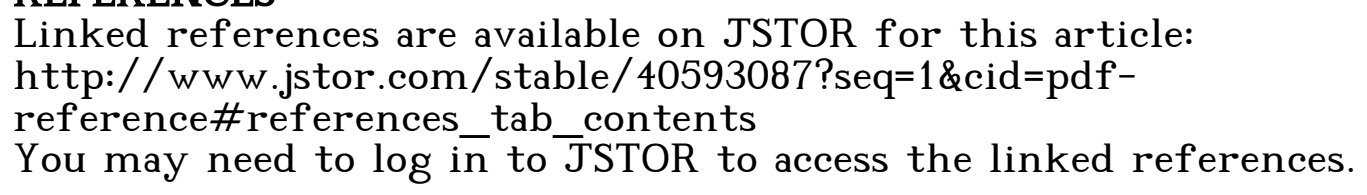

JSTOR is a not-for-profit service that helps scholars, researchers, and students discover, use, and build upon a wide range of content in a trusted digital archive. We use information technology and tools to increase productivity and facilitate new forms of scholarship. For more information about JSTOR, please contact support@jstor.org.

Your use of the JSTOR archive indicates your acceptance of the Terms \& Conditions of Use, available at https://about.jstor.org/terms 


\title{
RÉUSSIR DANS LES PECO EN INTRODUISANT L'ORIENTATION MARCHE : Une réflexion à partir du cas roumain
}

\author{
CORINNE ROCHETTE
}

Les pays Est-Européens représentent pour les entreprises occidentales des territoires attractifs. Parmi eux la Roumanie offre de nombreuses opportunités : des coûts avantageux et un marché très dynamique. Les entreprises désireuses de développer une présence durable sur ces marchés sont plus à même d'adopter des actions tournées vers la mise en ouvre des dimensions qui constituent l'orientation marché. Une étude exploratoire conduite auprès de responsables d'entreprise occidentales implantées en Roumanie révèle l'existence d'une forme de pré-orientation marché, elle permet de mettre en évidence les blocages d'ordre comportemental et culturel.

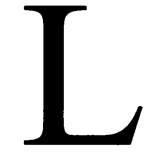

es PECO, nos très proches voisins, découvrent depuis plus de quinze ans les principes de fonctionnement de l'économie de marché. Ces territoires sont de plus en plus convoités par les entreprises étrangères en particulier Ouest-européennes en raison des opportunités qu'ils offrent à moins de 3 heures de vol de leur marché domestique. Dans un premier temps, celles-ci ont vu dans ces pays les avantages liés à une délocalisation de leur production, certains les accusant de vouloir en faire les ateliers de l'Ouest. Plus récemment elles se sont tournées vers des logiques d'implantation commerciale et de développement d'une présence durable. Certaines de ces entreprises adoptent à l'Ouest des logiques de marché élaborées et s'emploient à mettre en pratique les principes de l'orientation marché. La configuration particulière des marchés Est-européens peut les conduire à adopter sur ces marchés des logiques plus primaires pour un résultat plus immédiat. Cependant, les actions engagées montrent que, pour la plupart des grandes entreprises occidentales, ces territoires représentent des pays sur lesquels elles entendent se fixer durablement. Aussi, l'objectif de cet article est de discuter de l'intérêt et des difficultés des entreprises occidentales à introduire dans leur structure roumaine les composantes de l'orientation marché. Après avoir replacé la Roumanie dans le contexte des autres pays Est-européens, nous présenterons une typologie des logiques d'implantation. Nous montrerons, après avoir exposé les grands principes de l'orientation marché, que dans ces logiques peuvent être trouvées les dimensions plus ou moins affirmées de cette orientation. Nous exposerons enfin les principaux résultats d'une étude qualitative conduite auprès de managers d'entreprises occidentales implantées en Roumanie afin de comprendre leur mode de fonctionnement et les éventuels facteurs de blocage au développement d'une véritable orientation marché, enfin nous présenterons quelques pistes pour les dépasser.

Corinne Rochette est Docteur en sciences de gestion; elle est membre du GREGOR de l'IAE de Paris 1 et enseignante de l'Université d'Auvergne. Ses travaux portent sur l'orientation marché et le marketing relationnel. Elle $s$ 'intéresse aux pratiques marketing dans les pays en transition et à la place donnée au marketing dans les PME. Contact : corinne.rochette@u-clermont1.fr

Nous tenons à remercier les relecteurs pour leurs commentaires et suggestions ainsi que pour l'intérêt qu'ils ont porté à ce travail. 


\section{Les PECO : des marchés attractifs en construction}

Les pays d'Europe Centrale et Orientale présentent des situations contrastées même si l'on peut identifier des particularités communes. Si l'on retient comme critère de comparaison l'état du processus de transformation vers un fonctionnement de marché, trois catégories peuvent être identifiées : les pays ayant intégré l'union Européenne le $1^{\text {er }}$ mai 2004, ceux qui la rejoindront probablement en 2007 (Roumanie, Bulgarie) et ceux dont la transformation reste un lointain objectif (Ukraine, Moldavie, Biélorussie...). Les PECO ont longtemps été délaissés par les entreprises occidentales en grande partie en raison de leur instabilité macro-économique et financière. L'amélioration des indicateurs économiques (progression du pouvoir d'achat, taux de croissance annuel supérieur à $4 \%$, inflation maîtrisée, taux de chômage en baisse), du cadre de développement des affaires (privatisations, lutte contre la corruption, mesures en faveur des investisseurs étrangers, investissements et développement des infrastructures) en font désormais des zones présentant un véritable intérêt économique, avec de réelles opportunités, pour les entreprises occidentales, comme en attestent les importants flux d'investissements enregistrés au cours des trois dernières années. Le tableau 1 présente les principales données économiques de quatre de ces pays pour l'année 2005.
La présence d'entreprises étrangères dans les pays de la première catégorie remonte à plus de 10 ans et est conjointe aux vagues de privatisations. Leur arrivée a contribué au développement de nouvelles activités en particulier dans le domaine des services (grande distribution, banque, assurance) et à la rationalisation de certains secteurs (textile en Pologne, mécanique en Hongrie, automobile en Slovaquie). Pour ces pays, le paysage concurrentiel se fixe progressivement. Pour les pays de la deuxième catégorie, pour lesquels l'adhésion devient imminente, les problématiques se révèlent être quelque peu différentes. Ce n'est que très récemment (début des années 2000, même si la présence française en Roumanie remonte à 1991 avec Alcatel) que les entreprises étrangères ont découvert l'intérêt de ces territoires. Les investisseurs étrangers ont dans un premier temps été attirés par les opportunités offertes par les privatisations, l'accès à une main d'œuvre bon marché et qualifiée, plus récemment par la progression de la demande de biens et services (largement soutenue par des revenus issus de l'économie parallèle). En Roumanie et Bulgarie, de nombreux secteurs d'activité sont naissants (grande distribution, tourisme, hôtellerie économique, conseil, services bancaires et financiers, assurance) ou en restructuration (BTP, énergie, agroalimentaire). Le développement des premiers est impulsé par l'arrivée de grandes entreprises étrangères (à l'exemple de la grande distribution en Roumanie). En Bulgarie, la présence d'entreprises françaises reste modeste (77 implantations recensées à la fin de l'année 2005, dont la moitié dans les secteurs des services et des biens d'équipements professionnels). La Roumanie

Tableau 1

Indicateurs statistiques clés pour l'année 2005

\begin{tabular}{l|c|c|c|c}
\hline & Hongrie & Pologne & Roumanie & Bulgarie \\
\hline Population en millions & 10 & 38,1 & 21,7 & 7,7 \\
\hline PIB en milliards d'euros (€) & 124,2 & 364 & 78 & 21,4 \\
\hline Croissance réelle du PIB en \% & 3,9 & 3,2 & 4,1 & 5,5 \\
\hline Inflation en \% & 3,6 & 2,1 & 8,6 & 6,5 \\
\hline Chômage en \% de la population active & 7,3 & 17,6 & 5,9 & 10,1 \\
\hline IDE flux entrants en milliards d'euros $(€)$ & 4 & 10,3 & & 2,11 \\
\hline PIB/tête & $12420 €$ & $8031 €$ & $3590 €$ & $2771 €$ \\
\hline
\end{tabular}

Nota Bene : Pour les conversions entre Dollar et Euro nous avons utilisé le cours clôture des marchés du 30 décembre $2005(1$ Euro $=1,18450)$.

Sources : Banque mondiale, Mission économique des ambassades de France, Eurostats. 
qui fut le premier pays de l'Est à établir des relations officielles avec la communauté européenne en 1974 paraît être plus attractive. Cette attractivité s'explique par la taille de son marché (un des plus importants d'Europe de l'Est avec 21 millions d'habitants et plusieurs villes de plus de 600000 habitants) et par la persistance d'une certaine culture latine que les années de communisme n'ont pas gommée. Cette particularité semble atténuer les nombreux handicaps si l'on en croit la dynamique d'implantation des entreprises étrangères, plus particulièrement françaises, leur " enthousiasme " à aborder ce marché et à s'intéresser au consommateur roumain avide de dépenses et brimé par des années de pénurie.

\section{Les logiques d'implantation en Roumanie}

Au 31 décembre 2005, selon l'Agence Roumaine pour les Investissements Étrangers, la France occupait le $4^{\mathrm{e}}$ rang des investisseurs étrangers en Roumanie. La présence française s'est développée au travers d'une forte participation aux privatisations et s'est construite autour de grands groupes français tels que: Orange, Renault-Dacia, Société Générale-Banque Roumaine de Développement, Lafarge-Romcim. Tous les secteurs d'activité sont concernés, seul le secteur agricole, qu'il s'agisse de production ou d'industries de transformation est marqué par une sous-représentation malgré la présence de Danone, Limagrain, Cristal Union, Serve (vin). Le développement d'une présence sur le marché roumain répond à deux grands types de logiques.

- Une logique de coût : bénéficier de coûts avantageux, en particulier des faibles coûts d'une main d'œuvre présentant un bon niveau de qualification technique.

- Une logique de marché : conquérir le marché intérieur et tirer partie de sa dynamique de développement.

\section{La logique de coût}

On retrouve la logique de coûts assez naturellement dans des secteurs à faible valeur ajoutée et fortement utilisateurs de main-d'œuvre comme le textile. Les entreprises délocalisent leur production ou en sous-traitent une partie (on a utilisé l'expression d' "ateliers manufacturiers" pour qualifier cette pratique) afin de tirer partie de ces avantages économiques immédiats (2). La production est réexportée vers les zones de distribution telle que
l'Europe de l'Ouest. La réduction des coûts permet d'accroître les marges dégagées. Une partie de la production peut être utilisée pour une diffusion locale afin de tester la réceptivité du marché et de préparer le terrain pour une future commercialisation du produit à plus grande échelle lorsque le marché sera mûr (pouvoir d'achat supérieur, nouvelles habitudes de consommation). Cependant, la logique de coût s'inscrit dans un espace temps restreint. Elle présente une importante limite: la progression des salaires en Roumanie conduit les entreprises, en raison de la diminution et la perte de l'avantage initial, à envisager une délocalisation vers d'autres zones où la main d'œuvre est moins coûteuse. Les PMI semblent donc plus à même d'y avoir recours en raison de leur flexibilité, de leur facilité à redéployer rapidement leurs facteurs de production.

La logique de coût est principalement retenue par des entreprises dont la combinaison productive comporte une part importante de travail. On peut de ce point de vue s'interroger sur l'intérêt de la délocalisation pour les industries de service, par nature fortement utilisatrices de main d'œuvre. L'essentiel de leurs coûts est constitué par le coût du travail. La délocalisation par les entreprises italiennes de leurs plates-formes téléphoniques en Roumanie en est une illustration. Les recherches ont montré à travers le concept de servuction que la création du service provient en grande partie de la capacité du personnel à répondre aux exigences du client. La qualité du service généré est intimement liée à l'assimilation par les salariés du principe de "satisfaction du client"(7). Dans un pays où le concept même de client est récent, l'intérêt porté à la satisfaction du client est faiblement diffusé, ce qui peut être la limite au développement d'une activité de service. Les salariés sont plus rompus à exécuter les procédures définies par une autorité. Aussi, les entreprises de service susceptibles de tirer profit des coûts de main d'œuvre avantageux, sans pour autant altérer trop fortement la qualité du service délivré, sont celles pour lesquelles le processus de création de service est fortement standardisé (restauration rapide : Mc Donald's) et permet la définition de procédures simples. Dans cette perspective, la création de valeur se joue plus sur les aspects organisationnels qu'humains, plus sur le "savoir appliquer " que sur le "savoir interpréter", ce dernier exigeant de la part du salarié une véritable orientation client.

Si la logique de coûts est centrée sur l'optimisation de la combinaison de production, la logique de marché répond à la volonté de développer la présence commerciale. 


\section{La logique de marché}

Le marché roumain dans de nombreux secteurs est naissant. L'univers de consommation du consommateur Est-européen a longtemps été marqué par la rareté ; le simple fait de pouvoir se procurer le produit (le trouver et pouvoir l'acheter) semble le satisfaire. Dans un premier temps, les entreprises occidentales peuvent ou ont pu se contenter de sélectionner et commercialiser certains produits de leur portefeuille. La logique de marché est alors plus une logique de chiffre d'affaires. Des produits en fin de cycle de vie sur leurs marchés domestiques peuvent trouver une seconde vie à l'image, il y a quelques années, de la Renault 12 produite par le constructeur roumain Dacia, dans l'esprit du cycle de vie international du produit. Mais aujourd'hui, le consommateur des économies en développement accepte de moins en moins d'être celui à qui l'on offre d'acheter les modèles dépassés qui ont achevé leur vie sur les marchés matures et saturés. Aussi, dans une perspective de développement d'une présence commerciale durable, l'adaptation de l'offre et l'innovation s'avèrent judicieuses. Elles sont les révélateurs de l'adoption par les entreprises d'une logique de marché durable et exigent que les entreprises étrangères améliorent leur connaissance du consommateur.

La logique de marché peut être assimilée dans sa forme primaire à une simple logique de commercialisation, elle exige la présence d'espaces de vente pour toucher le consommateur final. La faible structuration du commerce en Roumanie présente des handicaps et des opportunités pour aborder ce marché. La disparition des magasins d'État s'est traduite par une explosion du petit commerce privé et indépendant. Pour une entreprise désireuse de commercialiser ses produits à grande échelle la tâche s'avérait délicate de parvenir à diffuser son produit sur l'ensemble du territoire. Le développement des grandes et moyennes surfaces impulsé par les grands groupes occidentaux (Métro, Carrefour) renforce les chances de succès commercial pour les entreprises de production. Ces grandes et moyennes surfaces densifient leur présence. Elles maîtrisent les règles du merchandising (l'art de mettre en valeur les offres et d'optimiser les ventes) et permettent d'améliorer la connaissance du comportement des consommateurs roumains à travers l'analyse des remontées caisse (types de produits achetés, quantités achetées, panier moyen...). L'apparition des premières cartes de fidélité, qui permettent le recoupement des données comportementales et socio-économiques pour une segmentation de la clientèle, va dans le même sens, celui de l'amélioration de l'information sur les clients et leur comportement. Cette évolution permettra de passer d'une logique de marché centrée sur l'écoulement de la production à une logique de création de valeur, de recherche plus poussée de la satisfaction du consommateur et elle va de pair avec le développement de compétences marketing. Par ailleurs, l'extension de réseaux de distribution dédiés tels que les réseaux de franchise, outre l'écoulement des offres, contribue à sensibiliser le personnel à l'intérêt du client et à des méthodes de travail éprouvées, grâce en partie à un management de proximité. Le tableau 2 présente une typologie des entreprises attirées par le marché roumain s'articulant autour des deux grandes logiques (coûts et marché).

La présence des entreprises étrangères sur le territoire roumain répond à des motivations variées. "Les maximisateurs " voient dans l'implantation sur ces territoires le moyen d'optimiser la combinaison des facteurs de production. Pour ces chasseurs de coûts, la logique est avant tout industrielle et s'intègre dans un espace temps réduit. "Les envahisseurs" perçoivent tout l'intérêt d'accompagner le développement de la consommation de masse en proposant leur offre. Cette deuxième catégorie recouvre deux types d'attitudes. Certaines entreprises ont un comportement opportuniste : vendre leur production en l'état. Pour elles, seul compte le chiffre d'affaires (logique de marché primaire). Pour d'autres, les actions s'inscrivent dans une stratégie d'ancrage et sont destinées à poser les fondements d'une réussite pérenne (logique de marché élaborée).

La figure 1 présente une schématisation de ces logiques.

Le marché roumain offre des possibilités dans le cadre d'activités centrées sur des logiques de coûts, même si l'intérêt se révèle éphémère. Les logiques de marché permettent, elles, d'évoluer vers un développement plus durable et s'accompagnent d'une prise en compte de la fonction marketing comme fonction stratégique.

\section{D'une logique de marché primaire ò une logique de marché élaborée : la place de la fonction marketing et de l'orientation marché pour un développement pérenne}

Dans un premier temps, l'ouverture des marchés, se traduit par la diffusion de produits et services " occidentaux", des nouveautés recherchées pour elles-mêmes par le consommateur local. Les entreprises 
Tableau 2

Une typologie des entreprises attirées par le marché roumain

\begin{tabular}{|c|c|c|c|c|c|}
\hline & \multicolumn{2}{|c|}{ « Les maximisateurs » } & \multicolumn{3}{|c|}{ « Les envahisseurs » } \\
\hline & $\begin{array}{c}\text { Type } 1 \\
\text { Logique de coût }\end{array}$ & $\begin{array}{c}\text { Type } 2 \\
\text { Logique de coût et } \\
\text { d'expérimentation } \\
\text { marché }\end{array}$ & $\begin{array}{c}\text { Type } 1 \\
\begin{array}{c}\text { Logique de marché } \\
\text { instantanée }\end{array}\end{array}$ & $\begin{array}{c}\text { Type } 2 \\
\text { Logique de marché } \\
\text { à terme }\end{array}$ & $\begin{array}{c}\text { Type } 3 \\
\text { Logique de marché } \\
\text { pérenne }\end{array}$ \\
\hline $\begin{array}{l}\text { Quel(s) } \\
\text { objectif(s) } \\
\text { pour } \\
\text { l'entreprise? }\end{array}$ & $\begin{array}{l}\text { Tirer profit des } \\
\text { avantages que la } \\
\text { Roumanie offre en } \\
\text { termes de coûts. }\end{array}$ & $\begin{array}{l}\text { Contrôler les coûts } \\
\text { et expérimenter la } \\
\text { réceptivité à la } \\
\text { production offerte. }\end{array}$ & $\begin{array}{l}\text { Tirer profit des } \\
\text { tendances } \\
\text { émergentes de } \\
\text { consommation et } \\
\text { des évolutions de } \\
\text { l'environnement } \\
\text { socio-économique. }\end{array}$ & $\begin{array}{l}\text { Développer une } \\
\text { présence solide. } \\
\text { Approche globale } \\
\text { du marché }\end{array}$ & $\begin{array}{l}\text { Se positionner, } \\
\text { conforter la position } \\
\text { et affronter la } \\
\text { concurrence. } \\
\text { Approche } \\
\text { segmentée du } \\
\text { marché }\end{array}$ \\
\hline Optique & Optique production & $\begin{array}{l}\text { Optique production } \\
\text { et vente }\end{array}$ & $\begin{array}{l}\text { Optique } \\
\text { commerciale }\end{array}$ & $\begin{array}{l}\text { Optique produit : } \\
\text { (adapter) }\end{array}$ & $\begin{array}{l}\text { Optique client } \\
\text { (innover) }\end{array}$ \\
\hline Priorités & Réduire des coûts & $\begin{array}{l}\text { Réduire des coûts, } \\
\text { Écouler (tester) la } \\
\text { production }\end{array}$ & $\begin{array}{l}\text { Développer la } \\
\text { présence } \\
\text { commerciale }\end{array}$ & $\begin{array}{l}\text { Adapter l'offre et } \\
\text { développer le } \\
\text { marché local }\end{array}$ & $\begin{array}{l}\text { Segmenter la } \\
\text { demande et adapter } \\
\text { l'offre au(x) } \\
\text { segment(s) }\end{array}$ \\
\hline $\begin{array}{l}\text { Logiques } \\
\text { d'affaires }\end{array}$ & Logique de marge & $\begin{array}{l}\text { Logique de marge et } \\
\text { de chiffre d'affaires }\end{array}$ & $\begin{array}{l}\text { Logique de chiffre } \\
\text { d'affaires }\end{array}$ & $\begin{array}{l}\text { Logique de parts de } \\
\text { marché }\end{array}$ & $\begin{array}{l}\text { Logique de } \\
\text { satisfaction des } \\
\text { besoins de la } \\
\text { clientèle }\end{array}$ \\
\hline $\begin{array}{l}\text { Avantages } \\
\text { recherchés }\end{array}$ & $\begin{array}{l}\text { Coûts bas d'une } \\
\text { main d'œuvre } \\
\text { qualifiée, } \\
\text { Coûts des matières } \\
\text { premières et/ou } \\
\text { logistiques réduits. }\end{array}$ & $\begin{array}{l}\text { Coûts bas et } \\
\text { possibilité } \\
\text { d'accroître les } \\
\text { volumes de } \\
\text { production et les } \\
\text { ventes (les } \\
\text { débouchés locaux } \\
\text { comme bonus). }\end{array}$ & $\begin{array}{l}\text { Tirer profit de la } \\
\text { maîtrise du process } \\
\text { de fabrication, } \\
\text { Allonger la durée de } \\
\text { vie des produits. }\end{array}$ & $\begin{array}{l}\text { Se positionner } \\
\text { comme un offreur } \\
\text { de solutions, } \\
\text { être une tête de pont } \\
\text { vers d'autres zones. }\end{array}$ & $\begin{array}{l}\text { La satisfaction du } \\
\text { client et la } \\
\text { fidélisation comme } \\
\text { avantage } \\
\text { concurrentiel. }\end{array}$ \\
\hline $\begin{array}{l}\text { Entreprises } \\
\text { concernées }\end{array}$ & $\begin{array}{l}\text { Industries de } \\
\text { production et de } \\
\text { transformation, avec } \\
\text { technologie } \\
\text { maîtrisée et stable }\end{array}$ & $\begin{array}{l}\text { Industries de } \\
\text { production et de } \\
\text { transformation, } \\
\text { avec technologie } \\
\text { maîtrisée et stable }\end{array}$ & $\begin{array}{l}\text { Entreprise de } \\
\text { production de biens, } \\
\text { plus } \\
\text { particulièrement les } \\
\text { réseaux de franchise }\end{array}$ & $\begin{array}{l}\text { Plus } \\
\text { particulièrement les } \\
\text { entreprises de } \\
\text { services (banque, } \\
\text { hôtellerie) }\end{array}$ & $\begin{array}{l}\text { Toute entreprise } \\
\text { avec une } \\
\text { concurrence forte }\end{array}$ \\
\hline $\begin{array}{l}\text { Principale } \\
\text { limite }\end{array}$ & $\begin{array}{l}\text { Évolution (rapide) } \\
\text { des coûts à la hausse }\end{array}$ & $\begin{array}{l}\text { Évolution (rapide) } \\
\text { des coûts à la hausse }\end{array}$ & $\begin{array}{l}\text { Trouver des } \\
\text { distributeurs, ou } \\
\text { développer un } \\
\text { réseau de } \\
\text { distribution }\end{array}$ & $\begin{array}{l}\text { Connaître les } \\
\text { attentes et } \\
\text { comportements du } \\
\text { consommateur }\end{array}$ & $\begin{array}{l}\text { Connaître les } \\
\text { attentes et } \\
\text { comportements du } \\
\text { consommateur, } \\
\text { évaluer et renforcer } \\
\text { la satisfaction, } \\
\end{array}$ \\
\hline $\begin{array}{l}\text { Usage fait } \\
\text { de la } \\
\text { production }\end{array}$ & $\begin{array}{l}\text { Production } \\
\text { réexportée vers } \\
\text { d'autres pays }\end{array}$ & $\begin{array}{l}\text { Production exportée } \\
\text { et utilisée pour un } \\
\text { écoulement local ou } \\
\text { régional. }\end{array}$ & $\begin{array}{l}\text { Production destinée } \\
\text { au marché local }\end{array}$ & $\begin{array}{l}\text { Production destinée } \\
\text { au marché local }\end{array}$ & $\begin{array}{l}\text { Produit et/ou service } \\
\text { destinés aux } \\
\text { segments ciblés }\end{array}$ \\
\hline $\begin{array}{l}\text { Exemples } \\
\text { d'entreprises } \\
\text { françaises }\end{array}$ & $\begin{array}{l}\text { PMI : Mobilux qui } \\
\text { produit pour Ikéa et } \\
\text { Conforama, Union } \\
\text { textile de } \\
\text { Tourcoing, } \\
\text { Dedienne dans la } \\
\text { plasturgie, } \\
\text { Grands groupes : } \\
\text { Michelin }\end{array}$ & Danone, L'Oréal & $\begin{array}{l}\text { Renault (Clio), Yves } \\
\text { Rocher, ACCOR } \\
\text { (Ibis) }\end{array}$ & $\begin{array}{l}\text { BRD-Société } \\
\text { Générale, Carrefour, } \\
\text { Renault-Dacia } \\
\text { (Logan) }\end{array}$ & Orange \\
\hline
\end{tabular}

Source : Création personnelle (données extraites d'une étude réalisée par l'auteur sur les entreprises françaises présentes en Roumanie).

Remarque: Les deux premières colonnes intitulées «Type 1, logique de coût » et "Type 2, logique de coût et d'expérimentation marché » concernent, pour la première, les entreprises industrielles en rapport ou pas avec un donneur d'ordre et les PMI, pour la deuxième, les entreprises de production de produits de grande consommation qui souhaitent expérimenter le marché. Les trois colonnes suivantes se rapportent à la logique de marché. Elles renvoient aux entreprises qui utilisent le service comme une source d'avantage compétitif et à celles présentes dans le secteur des services. 
Figure 1 : Schématisation des logiques d'implantation

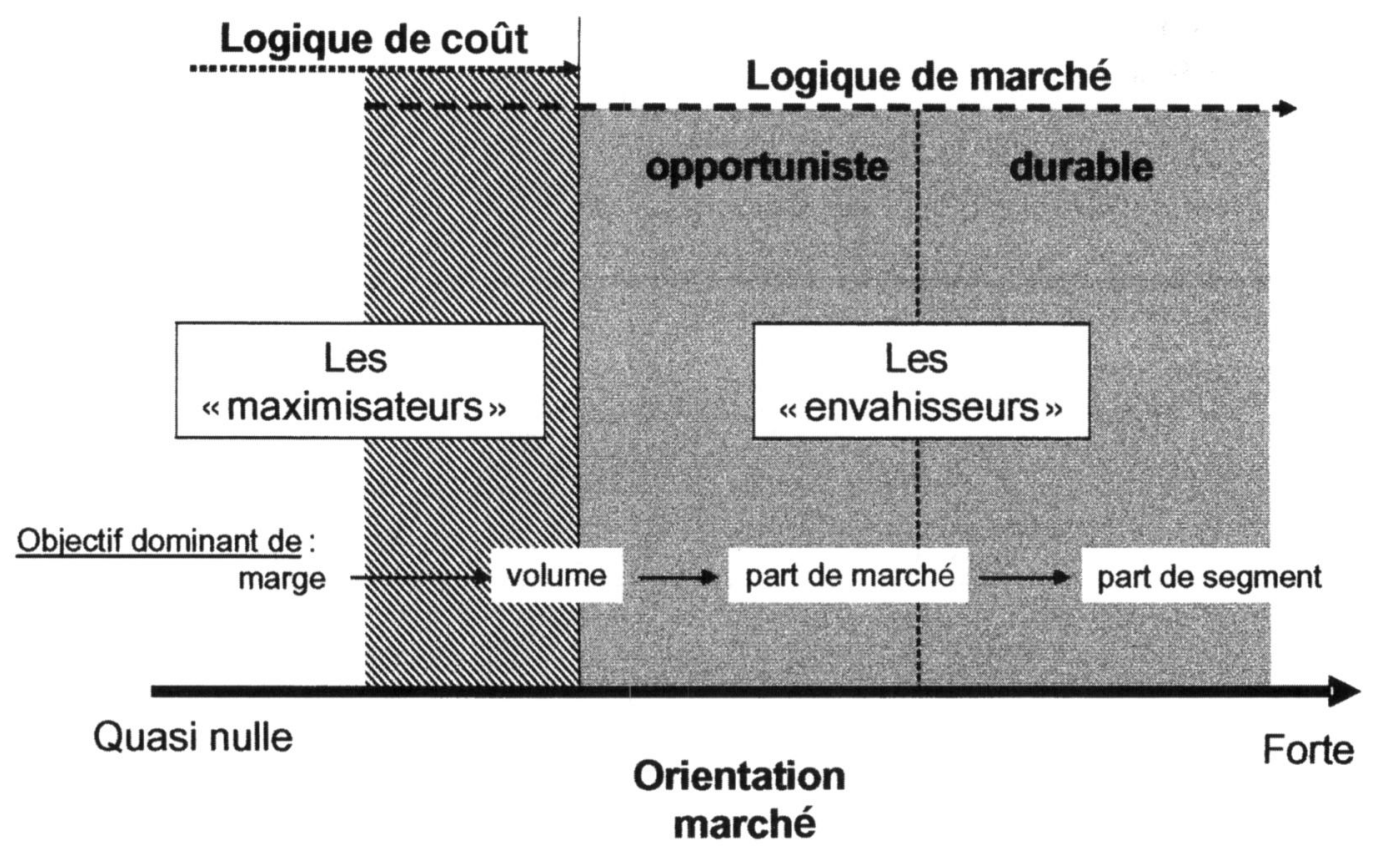

("envahisseurs de type 1 ") se contentent de commercialiser certains éléments de la gamme, leurs préoccupations concernent le dimensionnement de l'offre et la promotion : trouver les distributeurs et faire connaître (communication) leur produit ou service. Dans un deuxième temps, l'étoffement de l'offre, le renforcement de la concurrence, l'évolution à la hausse des exigences des consommateurs nécessitent d'adapter les offres (" envahisseurs de type 2 "). Le consommateur devient sensible au rapport qualité-prix en raison du renforcement de son choix. Il peut être pertinent à ce stade, pour les entreprises, d'envisager une combinaison logique de coûts/logique de marché. Le développement d'une orientation fournisseur apporte des réponses aux exigences de contrôle des coûts et d'adaptation des produits. La fonction marketing doit apporter une meilleure connaissance des attentes du consommateur et proposer des pistes quant aux adaptations nécessaires, cependant elle exige une maîtrise des outils d'étude et d'analyse du marché, des compétences qui aujourd'hui font défaut sur le marché roumain en raison de la récence de l'enseignement du marketing et de ces techniques dans les programmes de formation universitaires. Le dernier stade se caractérise par une intensité concurrentielle forte. Un contexte qui exige de l'entreprise ("envahisseurs de type 3 ") de parvenir à proposer une valeur supérieure au consommateur afin de se démarquer de la concurrence. La connaissance fine de la clientèle (orientation client), des offres des concurrents (orientation concurrent), la recherche de nouvelles solutions technologiques

(orientation technologie) sont les bases de la mise en place d'une stratégie de segmentation de la demande. La fonction marketing se concentre sur le repérage des segments les plus intéressants, le développement d'offres ciblées. La proposition d'une valeur supérieure devient un outil de fidélisation dans un contexte où les sollicitations au départ (du client) sont nombreuses. C'est à ce stade que le déploiement de stratégies de management de la relation client (CRM) prend toute sa consistance. Le système d'information devient un outil particulièrement stratégique.

Les entreprises occidentales présentes sur le marché roumain se trouvent face à un marché en structuration. La concurrence dans la plupart des secteurs y est encore globalement faible. Leur objectif est la conquête du marché, c'est-à-dire d'amener les consommateurs à acheter les produits et/ou les services en facilitant l'accès à l'offre (multiplication des points de vente, offre de crédit). De façon générale, leur approche ne constitue pas à proprement parler une illustration de l'orientation marché (dans sa complétude) pourtant adoptée sur leurs marchés domestiques. Ainsi, dans le secteur de l'hôtellerie-restauration le groupe ACCOR aborde la Roumanie avec prudence à partir du déploiement d'une seule catégorie d'offre : le produit Ibis (produit intermédiaire dans la gamme). La stratégie de marché élaborée adoptée sur son marché domestique (segmentation) n'est pas reprise. La raison réside en partie dans le déficit de connaissance des clients potentiels et de leur comportement. Le marché de 
l'hôtellerie en Roumanie a longtemps été et est encore largement un marché constitué d'une clientèle d'affaires, une activité couverte par les hôtels d'État (Hôtels Continental). L'hôtellerie en tant que marché de masse est nouvelle. Par ailleurs, elle est un marché sur lequel la création de valeur a largement pour origine le comportement du personnel et sa capacité à accueillir le client, à comprendre et satisfaire ses attentes. Le déficit de culture client des salariés roumains est particulièrement handicapant. Le développement d'une culture client c'est-à-dire de la recherche de l'intérêt du client puis dans un deuxième temps d'une orientation client (culture client à laquelle s'ajoute un système d'information centré client) sont des étapes préalables pour envisager le développement d'une véritable orientation marché, garantir l'homogénéité du concept Ibis et développer des atouts qui permettront d'affronter la concurrence future dans de bonnes conditions.

Les choses évoluent vite, le groupe ACCOR a ouvert le $1^{\text {er }}$ septembre 2006 un véritable hôtel "Amiral " dans le quartier des affaires à Bucarest ; le "Novotel Bucharest City Centre ». Ces changements conduisent à revoir la catégorie à laquelle l'entreprise peut être affectée. Une entreprise peut donc passer rapidement d'une colonne à l'autre de la typologie présentée dans le tableau 2 .

\section{L'orientation marché : un déploiement pas à pas et la nécessité d'un ancrage de l'"état d'esprit marketing"}

On ne peut pas envisager une présence durable sur ces marchés sans s'intéresser au client. Le marketing de l'offre qui permet dans le cadre d'une logique de marché primaire de satisfaire les objectifs de chiffre d'affaires montre rapidement ses limites.

Le client et ses besoins évoluent, ses exigences changent. Les modifications d'attitudes et de comportements sont soutenues par le renforcement de la concurrence et avec elle du choix. Il ne s'agit plus d'appréhender le marketing comme "la conduite d'activités commerciales qui orientent le flux de biens et de services des producteurs vers les consommateurs" (1), mais comme la recherche de la satisfaction du client, une philosophie d'entreprise qui prend toute sa dimension (6).

Diffuser "l'état d'esprit marketing ", défini par Felton en 1959 (3) auprès de salariés qui en perçoivent difficilement la consistance (pour des raisons historico-culturelles), devient une priorité. Dans un environnement d'affaires où le concept marketing est récent et la présence de services marketing dans les organigrammes des structures locales relativement nouvelle, les pratiques n'ont pas eu le temps de se cristalliser. À l'Ouest, la satisfaction du client est encore souvent exclusivement perçue comme la mission principale et la raison d'être du service marketing, avec les limites qu'une vision restrictive implique car il faut avoir une vision large à l'heure où les efforts de l'ensemble de l'organisation doivent converger vers la satisfaction du client (10) (12). Les résistances à une vision client partagée par toute l'organisation sont importantes, et les "part time marketers " encore trop rares. Les structures adoptées à l'Est se sont développées dans un contexte vierge de tout $a$ priori quant à l'affectation de la mission de satisfaction du client au seul service marketing. Le déploiement de l'état d'esprit marketing à l'ensemble de l'organisation peut être envisagé dès le départ comme une opportunité à saisir. Le service marketing se présente alors comme un moyen de coordonner et d'orienter les énergies vers l'amélioration de la valeur créée pour le consommateur et sa satisfaction. Une étape dont la réussite exige de lourds investissements en particulier en matière de formation.

L'impulsion d'un état d'esprit marketing est la première étape au développement d'une philosophie d'affaires tournée vers le marché.

\section{Les autres dimensions de l'orientation marché}

Contextuellement la naissance de l'orientation marché résulte de l'accroissement de la compétitivité mondiale (9) et de la nécessité de rechercher, en tout point de la chaîne de valeur, l'avantage concurrentiel. Elle est caractéristique d'une logique de marché élaborée. L'orientation marché s'inscrit dans une forme de questionnement de l'impact de l'état d'esprit marketing (orientation client) sur la performance de l'entreprise. La publication de deux recherches en 1990, celles de Kohli et Jaworski et de Narver et Slater (5) (8) vont poser les bases de la définition du concept d'orientation marché. L'orientation marché est abordée dans ses dimensions culturelle (le client comme valeur centrale (14)) et comportementale (la production, la diffusion d'informations à l'ensemble de l'organisation et la réactivité (5)). Dans un premier temps, ce concept s'articule autour des orientations "client " et " concurrent". Ces deux dimensions seront par la suite complétées par une " orientation technologique " mettant en évidence l'importance de l'innovation en tant que facteur de satisfaction du client et de compétitivité. De nombreuses recherches montrent l'impact positif de l'orientation marché sur l'innovation. Plus récemment, en raison de l'intégration du pouvoir des distributeurs et des 
fournisseurs dans l'analyse du système marketing et de la mise en évidence des gisements de compétitivité dont ils peuvent être la source, les "orientation fournisseur" et " orientation distributeur" sont, elles aussi, perçues comme des manifestations d'une culture d'orientation marché. Le déploiement de l'orientation marché et son impact sur la performance de l'entreprise sont conditionnés à un certain nombre d'éléments : l'attitude des cadres dirigeants, la configuration organisationnelle (centralisation, formalisation, départementalisation en autres), la nature des relations entre les départements (système de récompense, niveau de connexion, de conflit) (4). Les caractéristiques de l'environnement peuvent favoriser ou annihiler l'adoption de comportements et/ou la diffusion d'une culture favorables (11). Il s'agit de l'hostilité et la complexité de l'environnement, du taux de croissance de la demande et de son incertitude, de l'intensité concurrentielle, de la turbulence technologique... La figure 2 présente une schématisation des composantes de l'orientation marché.

\section{Typologie des entreprises et dimensions de l'orientation marché}

On peut retrouver dans les types d'entreprises attirées par le marché roumain certaines dimensions de l'orientation marché. La présence des dimensions présentées dans la figure 2 n'est pas typique d'une logique de marché élaborée. On trouve dans les entreprises adoptant des logiques de coûts ou des logiques de marché primaires certaines de ces dimensions. Les "maximisateurs" sont conduits à développer une orientation fournisseur. Celle-ci est particulièrement évidente dans le cadre de la délocalisation d'une activité sous-traitée. Le fournisseur contribue directement à la satisfaction du consommateur. La valeur délivrée au client est dépendante de la qualité du produit et des composants, de la capacité des fournisseurs à remplir leurs engagements en termes de qualité, de délais et de volume dans un contexte de coûts réduits. L'orientation fournisseur prend corps dans la mise en place de relations partenariales (les actions de Danone envers les agriculteurs roumains vont dans ce sens). Ces relations peuvent devenir un avantage concurrentiel, limiter les comportements opportunistes des fournisseurs et préparer le terrain à une évolution de la logique d'affaires : d'une logique de coût dont on sait qu'elle s'avère éphémère vers une logique laissant une plus grande place à l'innovation.

Figure 2 : Une schématisation des composantes de l'orientation marché (adapté de Gotteland (4))

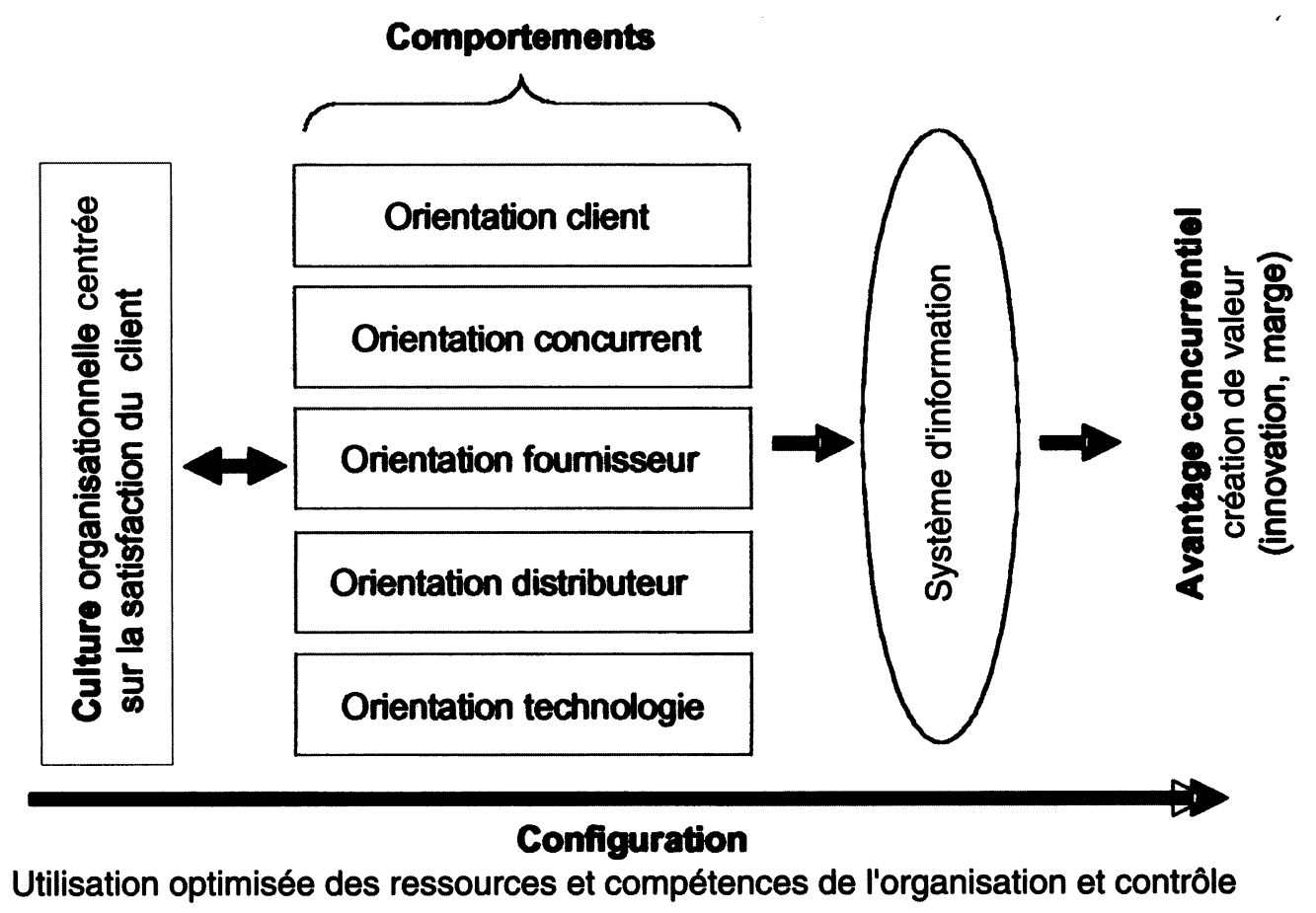

154 - Corinne ROCHETTE 
Dans le cadre d'une logique de marché primaire axée sur le chiffre d'affaires, les distributeurs en tant que prescripteurs ont une incidence directe sur le volume d'affaires et la part de marché. En contact avec le client, ils sont une source d'information sur les habitudes et comportements des clients, des informations qui, si elles sont recueillies et exploitées, facilitent l'adaptation des offres. C'est la perception de l'intérêt de ces informations qui conduira les distributeurs à adopter les comportements adéquats. Aussi, les blocages au développement de l'orientation marché dans les formes (fournisseurs et distributeurs) qui semblent les plus s'adapter actuellement aux caractéristiques du marché roumain des biens sont largement le fait de comportements et d'une culture défaillants. Précisons que, même en Roumanie, il est des entreprises qui adoptent des logiques de marché plus élaborées, ainsi le renforcement de la concurrence sur le marché de la téléphonie mobile a conduit Orange à développer une segmentation de la clientèle, et à mettre en place des outils CRM (orientations client, concurrent et technologie). Il paraît probable que les entreprises qui adoptent dans un environnement concurrentiel des logiques de marché plus sophistiquées travaillent à intégrer l'ensemble des dimensions de l'orientation marché, comme le montrent les exemples de Danone, ACCOR, Michelin, ou encore Renault, Carrefour sur leur marché domestique. Le tableau 3 relie les logiques d'affaires aux dimensions de l'orientation marché.
L'orientation marché dans sa forme la plus achevée s'inscrit probablement plus dans le cadre de logiques de marché. En ce qui concerne la logique de coût, on peut déceler l'intérêt de certaines de ses dimensions qui contribuent à une meilleure gestion de la chaîne de valeur externe. Si son intérêt existe bien, on peut s'interroger sur sa réalité et les problèmes posés par son déploiement sur des marchés où les comportements et la culture professionnels sont fortement imprégnés de ceux d'un autre modèle économique. Pour étudier ces deux aspects (réalité et problèmes), nous avons recueilli la perception des managers locaux des entreprises occidentales implantées en Roumanie.

\section{La réalité de l'orientation marché et les facteurs de blocage}

Une série d'entretiens semi-directifs a été conduite au cours de l'automne 2005 auprès de 10 directeurs marketing ou commerciaux d'entreprises occidentales. Les entreprises appartiennent au secteur de la production et/ou des services, elles présentent toutes une logique de marché, celle-ci est pour certaines combinée à une logique de coûts. Les entretiens ont été conduits en roumain puis traduits, intégralement retranscrits et ont fait l'objet d'une analyse de contenu manuelle et automatique (logiciel TROPES). Le guide d'entretien s'articulait autour trois thèmes : l'orientation marché dans sa globalité, le client, le marketing.

Tableau 3

Schématisation de la correspondance entre types d'entreprises et dimensions comportementales de l'orientation marchés

\begin{tabular}{|c|c|c|c|c|c|}
\hline \multirow[b]{2}{*}{$\begin{array}{l}\text { Logique } \\
\text { d'affaires }\end{array}$} & \multicolumn{2}{|c|}{ « Les maximisateurs » } & \multicolumn{3}{|c|}{ « Les envahisseurs» } \\
\hline & $\begin{array}{c}\text { Type } 1 \\
\text { Logique de coût }\end{array}$ & $\begin{array}{c}\text { Type } 2 \\
\text { Logique de coût et } \\
\text { d'expérimentation } \\
\text { marché }\end{array}$ & $\begin{array}{c}\text { Type } 1 \\
\text { Logique de } \\
\text { marché } \\
\text { instantanée }\end{array}$ & $\begin{array}{c}\text { Type } 2 \\
\text { Logique de } \\
\text { marché } \\
\text { à terme }\end{array}$ & $\begin{array}{c}\text { Type } 3 \\
\text { Logique de } \\
\text { marché } \\
\text { pérenne }\end{array}$ \\
\hline $\begin{array}{l}\text { Les probables } \\
\text { dimensions } \\
\text { comportementales } \\
\text { de l'orientation } \\
\text { marché }\end{array}$ & $\begin{array}{l}\text { - Orientation } \\
\text { fournisseur }\end{array}$ & $\begin{array}{l}\text { - Orientation } \\
\text { fournisseur } \\
\text { - Orientation } \\
\text { distributeur }\end{array}$ & $\begin{array}{l}\text { - Orientation } \\
\text { fournisseur } \\
\text { - Orientation } \\
\text { distributeur }\end{array}$ & $\begin{array}{l}\text { - Orientation } \\
\text { fournisseur } \\
\text { - Orientation } \\
\text { distributeur } \\
\text { - Orientation } \\
\text { consommateur } \\
\text { - Orientation } \\
\text { technologique }\end{array}$ & $\begin{array}{l}\text { - Orientation } \\
\text { fournisseur } \\
\text { - Orientation } \\
\text { distributeur } \\
\text { - Orientation } \\
\text { consommateur } \\
\text { - Orientation } \\
\text { technologique } \\
\text { - Orientation } \\
\text { concurrent }\end{array}$ \\
\hline
\end{tabular}




\section{Les dimensions de l'orientation marché en Roumanie : les principaux résultats}

Le discours des managers met en relief une certaine pré-orientation autour des composantes comportementales qui constituent l'orientation marché. L'analyse ne permet pas de mettre en évidence la présence nette de la dimension culturelle de l'orientation marché. L'impulsion d'une logique d'affaires orientée marché serait de ce point de vue plus le résultat de comportements effectifs; les dimensions comportementales précéderaient la dimension culturelle.

\section{- Connaître le client : une première étape vers l'orientation client}

Les discours mettent clairement en évidence une quasi " obsession " des entreprises à connaître leurs clients. La collecte et la transmission des informations sont une réalité. La structuration du système d'information autour du client permet de parler de pré-orientation client et pas seulement d'intérêt porté au client. Celle-ci pose des problèmes comme la communication entre les services. Pour les managers interrogés, la connaissance du client exige une implication forte du personnel à son contact et la mise en place de systèmes incitatifs. La mise en place de services client est aussi destinée à renforcer cette connaissance. La démarche de segmentation de la clientèle est très peu évoquée, l'approche du marché est plus globale, c'est donc plus de segmentation de l'offre dont il est question que de segmentation de la demande.

- Faire des offres compétitives qui dépassent les éléments qualité et prix en intégrant le service

La réussite sur le marché roumain dépasse la simple capacité à développer une offre performante (" dépasser la lutte sur le prix et le produit"). La proposition d'une offre compétitive en termes de qualité et de prix est considérée comme un acquis. On relève une volonté de développer les services associés au produit. Les "garanties" permettent de lever la défiance des clients rendus suspicieux par la qualité médiocre des produits commercialisés il y a quelques années. La "livraison à domicile " pour un pays où l'automobile n'a pas envahi tous les foyers est un plus important. Certains managers interrogés soulignent la "mission" d'éducation des consommateurs qu'assument les entreprises par l'intermédiaire de leur personnel ("le client ne sait pas comment utiliser le produit, on doit le lui apprendre, pour vendre "). Le personnel doit "être bien évidemment très bien formé ". Pour un des managers interrogés "l'entreprise crée plus de valeur par la qualité de ses représentants que la qualité de ces produits».

\section{- Développer des partenariats : une orienta- tion fournisseurs et distributeurs}

Les relations avec les autres acteurs de la chaîne de valeur reposent de plus en plus sur le partenariat. Il prend la forme de proposition de services, de programmes de formation. Certains managers évoquent la nécessité de développer des relations "win-win". La distribution apparaît comme une variable particulièrement critique ("nous devons accorder une grande attention aux points de vente pour que le produit soit disponible»).

\section{- Innover : une orientation technologique}

La dimension technologique apparaît à deux niveaux. Elle s'inscrit au niveau du partenariat développé avec les distributeurs (développer des outils et solutions leur permettant d'être plus efficaces) et, au niveau du client, de "son désir d'innovation". La performance de l'entreprise passe par le développement et la proposition de solutions technologiques novatrices. La nouveauté des produits ou services est perçue comme un moyen de retenir les clients. En l'état actuel, l'innovation est plus particulièrement dirigée vers certains types de clients, plus réceptifs à la nouveauté, ce qui tendrait à mettre en lumière la présence conjointe de l'orientation client et de l'orientation technologique, qui sont deux éléments constitutifs de l'orientation marché. L'encadré 1 présente l'exemple de la Logan comme la conciliation réussie entre la satisfaction du client et l'innovation.

La concurrence est très peu évoquée, même si les managers connaissent leurs concurrents, ils se préoccupent peu de leurs actions, ce qui traduit une absence de véritable orientation concurrent.

\section{Les facteurs de blocage au développement d'une philosophie d'affaires tournée vers le marché}

On peut relever les facteurs les plus importants de blocage au développement d'une logique d'affaires tournée vers le marché.

\section{- La culture client défaillante}

Le premier concerne le déficit de culture client pour les entreprises en contact avec le consommateur final. L'acheteur cède progressivement la place au client. Une 


\section{Encadré 1 : Concilier innovation et satisfaction du client : le cas de la Logan}

Se concentrer sur la proposition de valeur et faire preuve de créativité, tel a été l'objectif du groupe Renault-Dacia avec le lancement de la Logan de Dacia. Pour Y. Burnel, Secrétaire général du projet Dacia (direction des opérations internationales du groupe Renault) "La Roumanie connaît un véritable bouleversement de la consommation ", dans le domaine de l'automobile " les Roumains ont des goûts proches des nôtres même s'il existe un fossé entre leurs aspirations et leurs moyens " non encore comblé par le crédit à la consommation naissant. Le projet de la voiture à 5000 euros résulte de la volonté d'intégrer les dimensions économique, technologique, et les besoins du client dans la proposition d'une solution originale. La Logan est un produit qui permet de satisfaire un consommateur, en termes de désir de mobilité, qui dispose d'un pouvoir d'achat représentant moins de $35 \%$ de la moyenne du pouvoir d'achat des 25 pays de l'Union Européenne et de conforter la place du groupe sur la zone face à la concurrence, en particulier celle de Daewoo. Sur le plan technologique, elle comporte peu d'électronique pouvant ainsi être réparée par les garagistes roumains plus rompus à la mécanique "traditionnelle » qu'à la reconfiguration des ordinateurs de bord, par ailleurs sa robustesse en fait un véhicule adapté au réseau routier très endommagé.

évolution qui exige le développement de nouvelles compétences de nature commerciale chez le personnel : l'attention portée au client, l'écoute, la compréhension de ses attentes, le conseil, autant de qualités et d'attitudes à développer. Alors se pose le défi d'amener le personnel à considérer le client comme la raison d'être de l'entreprise, raison d'être qui a longtemps été le "Plan» imposé par une autorité centrale. Un des responsables de Gaz de France nous faisait part de son interrogation sur la façon d'amener les salariés de l'entreprise à intégrer la culture client : comment amener un Responsable client à prendre conscience qu'on ne peut pas recevoir un des clients les plus importants de l'entreprise dans un couloir entre deux portes ? L'évolution des comportements implique une diffusion de l'intérêt porté au client et une assimilation de la principale valeur qui lui est associée : la recherche constante de sa satisfaction.

\section{- Des systèmes d'information en structuration}

L'orientation marché apparaît comme un ensemble de compétences fondées sur la connaissance du marché ; dans ce contexte, l'information est une ressource stratégique conférant un avantage comparatif à ceux qui l'utilisent. Historiquement en Roumanie, la collecte et l'analyse de l'information étaient du domaine du renseignement (de l'intelligence), un domaine réservé aux militaires ; aussi pour les salariés des entreprises la collecte de données est une démarche nouvelle. Ils maîtrisent mal ou pas les outils qui lui sont associés (TIC). La collecte de données primaires est délicate. Les consommateurs restent méfiants envers le questionnement largement associé dans l'inconscient collectif aux pratiques des services de sécurité de leur pays. La compréhension des comportements se prête particulièrement à l'utilisation de techniques d'observation. Aussi, la fonction "études de marché " (encore peu développée sur place) portée par les services marketing nécessite de faire preuve de créativité quant aux techniques employées. Les sources d'informations secondaires se structurent, les données et statistiques sur l'évolution des marchés se développent et leur fiabilité s'améliore. Elles deviennent des sources de plus en plus fiables et exploitables. Mais le développement d'un système d'information n'est pas toujours possible ou s'avère délicat du fait d'une part d'une difficulté pour les salariés à appréhender l'intérêt, la valeur et la portée d'une information car l'information a longtemps fait l'objet de manipulation, et d'autre part du manque d'équipements.

La connaissance du marché est l'affaire de tous, le fruit d'une démarche collective (13). Il est de la responsabilité de la direction d'éduquer les salariés à cette pratique, de faciliter les échanges horizontaux afin de permettre la dissémination de "l'intelligence marché " dans l'organisation, une tâche délicate dans des structures encore très hiérarchisées et compartimentées et où la culture du secret prévaut.

\section{- Une intensité concurrentielle encore faible}

Par ailleurs, la création d'une valeur supérieure trouve sa justification dans un contexte concurrentiel agressif. Dans ces économies émergentes, la concurrence a récemment fait son entrée. Dans un environnement économique où, comme actuellement, la demande progresse fortement, la recherche d'un avantage sur la concurrence n'est pas une priorité ; en effet toutes les entreprises peuvent tirer profit de la dynamique du marché. La surveillance de la concurrence n'apparaît pas comme une nécessité : l'orientation concurrents est donc peu pratiquée. On peut estimer que cette attitude changera lorsque les managers découvriront l'impact des actions des concurrents sur la capacité de 
l'entreprise à atteindre ses objectifs, et donc les dangers à ignorer la concurrence.

\section{Quelques pistes pour dépasser les blocages}

La réussite des logiques de marché durable nécessite de parvenir à faire évoluer conjointement les comportements et la culture.

L'implication du top management dans le projet de réorganisation des modes de fonctionnement et de rénovation des pratiques est déterminante dans un pays où l'explicitation de l'idée d'initiative et d'animation est difficile à faire passer et où les compétences et la rémunération ont longtemps été deux choses déconnectées. Ce défi est prioritaire dans le domaine des services, qui est un secteur naissant. La Banque Roumaine de Développement (BRD - groupe Société Générale) affiche clairement dans son rapport annuel de 2004 que son objectif dans le domaine des ressources humaines est de développer les compétences et de motiver les équipes commerciales. Elle définit sa mission autour de l'adaptation de la ressource humaine aux nécessités du secteur bancaire; celle-ci implique la définition d'une politique de rémunération motivante et le développement de la formation professionnelle. La rémunération est un point clé, en particulier sur des marchés du travail tendus, tel que celui de la Roumanie où le taux de chômage est inférieur à $6 \%$, et dans la capitale de l'ordre de $3 \%$. Les roumains qui offrent des compétences commerciales et managériales sont très sollicités, voire "chassés " par les entreprises occidentales entrant sur le marché. En effet, ils ont une connaissance du marché et des mécanismes d'affaires locaux, ainsi que la maitrise des techniques de gestion occidentales. La rémunération est le moyen de fidéliser les compétences et d'espérer obtenir un retour sur les investissements en formation réalisés dans un contexte d'amplification de la mobilité des personnels formés. Dans le domaine de la grande distribution, Metro et Carrefour ont formé des salariés à de nouveaux métiers dont les compétences sont aujourd'hui convoitées par les nouveaux arrivants. Le groupe Renault-Dacia offre à ses salariés une rémunération supérieure à la moyenne du pays, mais aussi d'autres avantages auxquels les roumains sont sensibles : le repas de midi, un centre médical, des accès privilégiés au crédit. Les actions des entreprises occidentales en matière de formation et l'évolution de la politique d'éducation contribuent à créer un climat et des comportements propices à la diffusion de l'état d'esprit marketing.
L'accompagnement, qui est une forme de tutorat, permet lui aussi la diffusion et l'apprentissage d'une nouvelle logique d'activité. Les responsables de la Banque Roumaine de Développement (groupe Société Générale) considèrent que "la mise en place d'un binôme expatrié/manager local est un des meilleurs moyens d'insuffler une culture managériale aux cadres roumains", et cela d'autant plus que la culture latine des roumains favorise le dialogue. Les salariés roumains présentent une importante capacité d'apprentissage, comme le souligne la mission économique de l'ambassade de France à Bucarest : " la discipline et l'attitude au travail des roumains dans les entreprises étrangères sont satisfaisantes, notamment lorsqu'on prend le temps de leur expliquer la logique et le mode de fonctionnement de l'organisation". La transposition des méthodes de management de projet peut s'avérer une piste à explorer. Le management de projet se caractérise par le travail en petits groupes d'individus aux compétences et cultures techniques variées, par l'utilisation de méthodes d'apprentissage, par la définition d'objectifs qui peuvent être, par exemple, la recherche de la meilleure satisfaction possible du client, par l'intégration de contraintes économiques et temporelles ainsi que d'éléments auxquels le salarié doit être familiarisé pour gagner en productivité et en autonomie.

\section{Conclusion}

L'objet de cet article est de contribuer à une meilleure compréhension des problématiques de développement commercial sur les zones marquées par de profonds bouleversements et ruptures, et à replacer dans ce contexte l'intérêt du développement de l'orientation marché. La réussite durable de l'entreprise dépendra de sa capacité à développer des comportements et une culture tournés vers le marché, en dépassant les contraintes actuelles de pilotage auxquelles elles se trouvent confrontées, comme le manque d'informations ou le manque de savoir faire du personnel. L'étude exploratoire à partir d'une enquête auprès de managers roumains met en évidence l'existence d'une forme de pré-orientation marché : dimension stratégique, recherche de la satisfaction du client, innovativité, structuration des systèmes d'information, approche élargie de la chaîne de valeur. Si la présence de certaines dimensions comportementales de l'orientation marché a pu être identifiée tel n'est pas le cas de la dimension culturelle. Sur un plan purement académique la compréhension des dynamiques en œuvre peut permettre d'explorer l'articulation entre les dimensions comportementales et culturelles de l'orientation marché. Ce travail 
gagnerait à être complété par une mesure de la nature de la pré-orientation marché auprès des entreprises occidentales présentes en Roumanie afin d'affiner les typologies des logiques d'implantation. Sur un plan plus managérial, comme nous l'avons vu, la Roumanie est sans conteste un territoire attractif pour les entreprises étrangères sur lequel l'intensité de la concurrence est encore faible. Pour les entreprises désireuses de s'implanter et d'adopter une philosophie d'affaires tournée vers le marché, il convient dans un premier temps de se concentrer sur l'élément à la base du commerce : rechercher les moyens de favoriser l'accès du consommateur à l'offre. Pour ce faire, elles peuvent jouer soit sur la variable économique (logique de coût dominante), l'orientation fournisseur s'avère alors importante, soit sur le développement de la couverture du marché (l'orientation distributeur devient un point clé). Cependant, la réussite durable s'inscrit dans la capacité à développer chez les salariés les comportements et la culture adéquats et ce d'autant plus que la tertiarisation de l'économie qui accompagne la maturation de l'économie de marché exige une ressource humaine de qualité. L'éducation et la formation sont des éléments critiques. Par ailleurs, l'innovation en matière de services est aussi une piste à explorer : la proposition de crédit à la consommation, de paiements fractionnés ou différés bien que n'étant pas dans la culture roumaine peuvent s'avérer être des arguments de poids, la vente par correspondance ou la vente en réunion qui sied bien à la convivialité très latine de ce peuple peuvent permettre de combler la faiblesse des structures de distribution. Les actifs disposent de compétences techniques de qualité, en revanche les compétences de pilotage et managériales font encore défaut. Aussi, les implantations doivent s'accompagner dans un premier temps par une expatriation de salariés domestiques. Sur un plan plus global, l'orientation marché telle qu'elle est abordée sur le plan académique, où dans une version moins accomplie telle que certaines entreprises à l'Ouest s'efforcent de la mettre en place, n'a pas de véritable consistance en Roumanie. Le chemin pour en instaurer les fondations est encore long, la première étape réside dans la diffusion de l'état d'esprit marketing, une démarche pas à pas !

\section{Références}

(1) American Marketing Association (1948), Report of the definitions committee, Journal of Marketing, 13, 202.

(2) Berger S. (2006), Made in Monde, Paris, Éditions du Seuil.

(3) Felton A. (1959), Making the marketing concept work, Harvard Business Review, 37, 3, 55-65.

(4) Gotteland D. (2004), L'orientation marché, Paris, Éditions d'Organisation.

(5) Kohli A. K. et Jaworski B. J. (1990), Market orientation: the construct, research propositions and managerial implications, Journal of Marketing, 54, 1-18.

(6) Lambin J.J. (1999), Le marketing stratégique : du marketing à l'orientation marché, Paris, Ediscience international.

(7) Lovelock C., Lapert D. (2000), Marketing des services, Paris, Publi-Union.

(8) Narver C. J. et Slater S. F. (1990), The effect of a market orientation on business profitability, Journal of Marketing, 54, 20-35.

(9) Pras B. (1999), Les paradoxes du marketing, Revue Française de Gestion, septembre-octobre, 125, 99-111.

(10) Slater S. F. et Narver C. J. (1995), Market orientation and the learning organization, Journal of Marketing, 59, 63-74.

(11) Slater S. F. et Narver C. J. (1994), Does competitive environment moderate the market orientation performance relationship ?, Journal of Marketing, 58, 46-55.

(12) Ruekert R. W. (1992), Developing a marketing orientation : an organizational strategic perspective, International Journal of Research in Marketing, 9, 225-254.

(13) Sinkula J., Baker W. et Noordewier T. (1997), A framework for market based organizational learning: linking values, knowledge, and behavior, Journal of the Academy of Marketing Science, 25, 305-318.

(14) Webster Jr. F. E. (1992), The changing role of marketing in the corporation, Journal of Marketing, 56, $1-17$. 